\title{
Two Problems for Proportionality about Omissions
}

\author{
SARA BERNSTEIN ${ }^{\dagger}$ \\ (penultimate version; final version forthcoming in Dialectica)
}

\begin{abstract}
This paper argues that Dowe's implementation of the proportionality strategy can't solve a major problem for causation by omission, the problem of profligate omissions, for two reasons. First: the determinate/ determinable relationship that holds between properties like aqua and blue does not hold between negative properties like not aqua and not blue. Negative properties are those at stake in omissive causation. Second: proportionality misconstrues the nature of the problem to be solved.
\end{abstract}

Suppose that the gardener promises to water your plant while you are out of town, the gardener fails to water it, and the plant dies. Intuitively, the gardener's failing to water the plant is a cause of the plant's death. But the Queen of England also failed to water the plant, and the counterfactual "Had the Queen of England not failed to water the plant, the plant would not have died" is true. Thus a simple counterfactual test would lead us to classify the Queen of England's omission as a cause of the plant's death. This difficulty is known as the problem of profligate omissions: if we accept any omissions as causes, it seems that we must accept all omissions as causes.

How can we metaphysically distinguish the relevant omission or omissions from the irrelevant ones? A strategy under recent consideration is proportionality. ${ }^{1}$ The proportionality strategy employs the determinate/ determinable relationship to restrict which among many putative causes is the cause. The determinate/ determinable relationship is the special hierarchical relationship of ascending specificity that holds between properties like aqua and blue, and circular and shaped. The general idea of proportionality is that $c$ is a cause of $e$ only if $c$ has the right level of detail. If Sophie the bird is trained to peck at blue objects, and she is presented with a turquoise object at

\footnotetext{
† Department of Philosophy, Duke University, Durham, United States; Email: sara.bernstein@ duke.edu

${ }^{1}$ Dowe (2010) argues in favor of this strategy for omissive causation; Weslake (2013) and Sartorio (2010) consider this view but ultimately reject it for reasons different from mine. Shapiro and Sober (2012) argue against the strategy in general.
} 
which she pecks, there are multiple causal candidates for Sophie's pecking, at different levels of specificity: the object's being colored, the object's being blue, and the object's being turquoise. Applying proportionality to the case yields a single determinate--the object's being blue--as the cause of the pecking. Proportionality has mainly been used in the literature on mental causation to avoid widespread redundant causation of mental and physical properties ${ }^{2}$, but proportionality has also recently been applied to causation by omission in an attempt to divide causally relevant omissions from irrelevant ones. The idea is that only the determinate at the right level of specificity-for example, "the plant's not receiving an influx of water" - is the cause.

In this paper I argue that Dowe's (2010) execution of the proportionality strategy ${ }^{3}$ does not successfully resolve the problem of profligate omissions for two reasons: (i) the determinate/ determinable relationship can't be sensibly applied to negative properties, ${ }^{4}$ which are the sort of properties at stake in omissive causal claims; and (ii) proportionality misconstrues the problem of profligate omissions, and addresses a different problem instead.

Roadmap: In section 1, I present Dowe's proportionality solution to the problem of profligate omissions. In section 2, I set out the key features of the determinate/ determinable relationship, and show that the relationship doesn't apply to negative properties. In section 3 , I argue that this problem blocks the proportionality solution to the problem of profligate omissions. I also suggest that the proportionality strategy suffers from another difficulty: it solves a different problem than the one it purports to solve.

\section{The Proportionality Proposal}

Return to the problem of profligate omissions. Dowe holds that the determinate/ determinable relationship can be used to identify one of putatively many causes by

\footnotetext{
${ }^{2}$ Most prominently in Yablo 1992: 245-80.

3 Dowe's particular implementation of the proportionality strategy centers around the determinate/ determinable relationship.

4 The dialectic requires the assumption of genuinely negative properties for several reasons. First, they are the type of properties at stake in omissive causation. Second, the problem of profligate omissions affects the realist about omissions, and thus one who is unlikely to hold that the causal powers of negative properties derive from their positive features.
} 
holding that only the cause proportional to the effect is the actual cause.

Based on Yablo's (1992) proportionality constraint, the test for identifying a single determinate as the proportionate cause is counterfactual. Consider the claim that the gardener's failure to water the plant is the cause of its death. We can take the relevant negative properties $^{5}$ of the plant to be:

not watered by the gardener while humming, not watered by the gardener not watered by anyone

Dowe holds that the negative properties not watered by the gardener while humming and not watered by the gardener are determinates of the determinable not watered by anyone. The negative property not watered by the gardener while humming is not the cause of the plant's death, since had the gardener watered the plant but not been humming, the plant would not have died. Similarly, the property not watered by the gardener is not the cause of the plant's death, since had the plant been watered by the Queen of England (but not by the gardener), the plant would not have died. So neither determinate is the proportionate cause of the plant's death.

We then reapply proportionality to the property not watered by anyone by taking properties of the plant to be not receiving any water and not receiving an influx of any substance. Is the counterfactual "Had the plant not failed to receive an influx of any substance, it would have survived" true? No, for the plant could have received another substance, such as apple juice, and still died. But the counterfactual "Had the plant not failed to receive any water, it wouldn't have died" is true. So, according to Dowe, the plant's failing to receive water is the cause of its death. ${ }^{6}$

\footnotetext{
5 There are two ways to understand how a negative state of affairs can be causal. The first understanding permits the existence of negative properties, such as the plant's property not watered by anyone. The alternative understanding denies negative properties but admits failures of a property to obtain, such as the plant failing to have the property watered by anyone. The former takes negative properties to be the causal relata. The latter takes some sort of extra fact, such as the fact that the plant fails to have the property 'watered by anyone', as the relatum. I proceed with the former treatment-negative properties - for two reasons. First, the most well-known proponents of the proportionality strategy use such a treatment. Second, the latter treatment involves causal relata other than properties such as facts or propositions. That framework yields different and unique problems that are outside of the scope of this paper.

${ }^{6}$ Dowe admits that his solution yields the counterintuitive result that the gardener's failure to water the plant is not a cause of the plant's death.
} 


\subsection{Determinates and Determinables}

Let's take a closer look at the determinate/ determinable relationship upon which Dowe's proportionality strategy relies. Four features characterize this relationship: entailment of determinate to determinable, greater specificity of a determinate than its determinable, resemblance between determinates of the same determinable ${ }^{7}$, and incompatibility of determinates of the same determinable at the same level of specificity. ${ }^{8}$

First, entailment. Roughly, a property $\mathrm{x}$ determines a property $\mathrm{y}$ only if having $\mathrm{x}$ entails having y. Aquaness thus entails blueness. Another canonical example is squareness and shape: anything that instantiates the property square instantiates the property shaped.

Second, specificity. Instantiating the property aqua is a more specific way of instantiating the property blue. The direction of determination runs from specific to general: anything that has the specific property, such as aqua, also has the general property, blue. It is natural to think of specificity as a continuum of ascending detail. For example, what it is to be aqua is to be blue with a greater level of detail.

Third, resemblance between determinates of the same determinable. The property aqua resembles the property turquoise more than it resembles the property circular, in virtue of aqua and turquoise instantiating the same determinable blue.

Fourth, incompatibility of same-level determinates of the same determinable. Differently leveled determinates of the same determinable are compatible: turquoise is a determinate of blue, which is a determinate of colored. By entailment, all three properties coexist. But different determinates at the same level of specificity of the same determinable cannot be instantiated at once: something cannot be both turquoise and aqua at the same place and at the same time.

Arguably, what undergirds these features is that the determinate/ determinable relationship generates a genuine hierarchy of properties. There is a genuine hierarchy when the properties can be organized into a tree structure, with the most determinable

\footnotetext{
${ }^{7}$ When a property is the determinate of more than one determinable, comparative similarity judgments are difficult. Thus square might be said to resemble equilateral triangle as much as it resembles parallelogram. But it resembles both more closely than it resembles red.

${ }^{8}$ Here I follow Funkhouser (2006) in taking these four features to be canonical. I am not giving an analysis of the determinate/ determinable relationship, but I take these four features to be necessary conditions of the relationship.
} 
property as the highest node, and increasing determinacy as one descends the tree. The four key features can be framed in terms of this hierarchy, as follows:

(1) Entailment: Having a property located at one node of the tree entails the possession of all parent properties of that node. (For example, being aqua entails being blue; being blue entails being colored.)

(2) Specificity: If property $\mathrm{X}$ is at a child node of property $\mathrm{Y}$, then instantiating $\mathrm{X}$ is a way of instantiating Y. (For example, being aqua is a way of being blue and being colored.)

(3) Resemblance: Sibling nodes on the tree exhibit genuine resemblance relations. (For example, being aqua and being turquoise generate genuine resemblance between objects having those properties.) Moreover, greater determinacy generates closer resemblance. (For example, being aqua generates closer resemblance than being blue.)

(4) Incompatibility of determinates at the same level: If properties $\mathrm{X}$ and $\mathrm{Y}$ are tree siblings, then no object can instantiate both $\mathrm{X}$ and $\mathrm{Y}$ at the same place and at the same time. (For example, an object cannot exhibit the properties turquoise and aqua at the same time and in the same place.)

This hierarchy of properties suggests that determinates are more fundamental or "basic" than their determinables. ${ }^{9}$ Here are several reasons to think so. First, determinates generate greater objective resemblance between objects than their determinables. Two objects that are turquoise are more similar than two objects that are different shades of blue with respect to their color. And two objects that are circular are more similar than two objects that are differently shaped with respect to their shape. Genuine resemblance is a hallmark of fundamental or "natural" properties. Second, we need only fix the world's determinates to fix its determinables. Fixing an object's being turquoise and spherical ipso facto fixes its being blue and being shaped. Finally, it seems natural to hold

\footnotetext{
${ }^{9}$ For an opposing view, see Wilson (2012), who argues that determinates are not necessarily more fundamental than their determinables.
} 
that specific properties "carve nature closer to the joints" than general properties. Like the disjunctive property of something's being green or red, one might view the property blue as a disjunctive property of something's being aqua or turquoise or teal (or one of the many other determinates of blue). Presumably, the nondisjunctive, specific properties carve nature closer to its joints than the disjunctive ones..$^{10}$ As we'll now see, this hierarchy also provides a natural framework within which to evaluate the applicability of the relationship to negative properties.

\subsection{The Determinate/ Determinable Relationship and Negative Properties}

There are two ways that one can apply the determinate/ determinable relationship to negative properties: either by maintaining that the relationship matches that of positive properties (for example, by maintaining that not aqua is a determinate of not blue) or by reversing the direction of the relationship (for example, by maintaining that not aqua is a determinable of not blue). ${ }^{11}$ As I will now argue, neither option is tenable.

First, it is worth noting that negative properties do not exhibit differences in fundamentality parallel to those of positive properties. Consider a more fundamental property $^{12}$ and a less fundamental property: having a mass of two grams and being in the northern half of Nevada on a Tuesday. It is safe to assume that the property having a mass of two grams is more fundamental than the property being in the northern half of Nevada on a Tuesday: the property having mass yields more resemblance between objects than being in the northern half of Nevada on a Tuesday, is less disjunctive, and carves nature closer to its joints.

But the same isn't true for the negations of those properties: the property not having a mass of two grams does not yield more resemblance between its instantiators

\footnotetext{
${ }^{10}$ One need not be convinced that determinates are more fundamental than their determinables in order to accept that determinates and determinables form a tree structure. Similarly, one can accept the determinate/ determinable relationship but deny that it is based on a hierarchy. Here both are useful tools for illuminating the special nature of the determinate/ determinable relationship.

11 What distinguishes between negative and positive properties is a vexed issue. See Miller et al. (2013) for extensive discussion of this question. Here I do not make any claims about the relationship between negative properties and their descriptors, but assume, with Dowe, that we have a good enough grip on what negative properties are to proceed with the discussion.

${ }^{12}$ It is natural to view fundamental properties to be those posited by physics, i.e., Lewisian "natural" properties.
} 
than not being in the northern half of Nevada on a Tuesday. ${ }^{13}$ All else being equal, two objects are not more similar in virtue of having the former property rather than the latter. And neither negative property carves nature closer to the joints than the other. Applying this lesson to our previous example, two objects are not more similar if they both have the property not aqua as opposed to not blue. The property not aqua doesn't carve nature closer to the joints than the property not blue. Perhaps sharing many negative determinates might generate similarity, but not necessarily more similarity than sharing negative determinables.

These initial points suggest that negative properties do not naturally lend themselves to the same tree structure as positive properties. Since the hierarchical structure is central to the determinate/ determinable relationship in its familiar form, we should be pessimistic about the relationship's prospects for negative properties.

Let's consider whether the negative property pair not aqua and not blue exhibits the four features of the determinate/ determinable relationship. First, entailment. There is no entailment from not aqua to not blue: something's having the property not aqua doesn't necessarily guarantee that it has the property not blue. But the reverse holds: the general property not blue determines the property not aqua, for if something is not blue it cannot be aqua. Negating the properties retains entailment by reversing its direction.

Second, specificity. Is having the property not aqua a more specific way of having the property not blue? Intuitively, no. The entailment relation holds, but the relationship of more specific to less specific is absent.

There is a temptation to hold that, along with entailment, we can simply reverse the direction of the relationship, such that not blue is a more specific way of being not aqua. Call this idea "reverse specificity". A motivating example of reverse specificity is the following: suppose that my friend sends me to the paint store with instructions to buy paint that isn't aqua, so I return with turquoise paint. The friend complains that the paint is still blue. It seems fair for me to respond that the friend should have been more specific: she should have specified that the paint shouldn't be any shade of blue. ${ }^{14}$ This is prima facie evidence that having the property not blue is a more specific way of having

\footnotetext{
${ }^{13}$ One might try to argue that a property like not weighing more than one gram is more fundamental than not being in the northern half of Nevada based on the former's being intrinsic to its bearer. But one would need an independent argument for intrinsicness being a marker of fundamentality.

14 Thanks to Mark Balaguer for this example.
} 
the property not aqua.

Reverse specificity is especially tempting because, on the face of it, specificity is captured by the subset relation. According to this way of looking at specificity, property $x$ is more specific than property $y$ because the set of things that are $x$ is a subset of the set of things that are $y$. When I respond to the friend that she should have been more specific, I mean that she should have listed the set of paint colors - all paint colors that are blue - as the ones not to buy. Since there are fewer paint colors that are not blue than ones that are not aqua, it is tempting to view the former as a more specific property than the latter.

But the type of specificity involved in the determinate/ determinable relationship involves more than the subset relation. Being $\mathrm{x}$ is a more specific way of being $\mathrm{y}$ in virtue of its being y with a greater level of detail. For positive properties, this feature is captured by the fact that entailment and specificity hang together: to have the property aqua is not merely to belong to the set of things that are blue, but to have the property blue in a greater level of detail-to have a property that is relevant to blueness.

Alone, the subset relation isn't sufficient to establish such a relationship of differential detail because negative properties have many irrelevant determining properties. While ways to have the property blue are both limited in number and limited to properties relevant to blueness (such as having the properties aqua or turquoise), there are potentially infinite ways to have the property not blue, and most are irrelevant to color. For example, consider the following properties which, when possessed, presumably also guarantee possession of the property not blue: being a calcium flame and being the number 17. Being orange and being yellow are more specific ways of being not blue, but being the number 17 has nothing to do with being or not being blue, even if it is a way of determining possession of the property not blue. ${ }^{15}$

Specificity and entailment hang together for positive properties because positive determinates guarantee relevance to their positive determinables. But entailments between negative properties do not guarantee relevance between the entailed properties indeed, there are many irrelevant entailments. That entailment and specificity come apart

\footnotetext{
${ }^{15}$ One might hold that context determines the relevant contrast class. For example, a contrast class of colors is invoked when one speaks of something being "not blue," implying a relevant property such as blue or yellow, thus preventing the specificity problem. For example, if someone says of wallpaper, "That is not blue", it is presumed that the contrast class is only other colors (red, yellow) and not other things (being coffee, being a tree). But a correct analysis of determinates and determinables must float free of context and contrast class. Positive determinables do not require a contrast class for the relevance relation to hold.
} 
for negative properties undermines the foundation for straightforwardly ascending or descending detail between negative properties.

Here is another way to put the point. We can think of the property blue as equivalent to the disjunction of its more specific determining properties, i.e., turquoise or aqua or navy. Turquoise and aqua and navy are relevant to blue. But the disjunction of properties that entail not blue includes properties irrelevant to blueness: being a calcium flame and being the number 17 are part of the disjunction (as are more relevant properties like orange and green.) Having the property aqua guarantees having a property relevant to the property blue, but having the property not aqua doesn't guarantee having a property relevant to not blue (and vice versa.) ${ }^{16}$ The result of the separability of entailment and specificity for negative properties is that the key feature of the determinate/ determinable relationship — having a property with greater detail — isn't captured by reversing the direction of specificity. ${ }^{17}$

We can now turn to the third feature of the determinate/ determinable relationship: resemblance. Sibling negative determinates are not related by resemblance. If one object has the property not turquoise and the other object has the property not aqua, the objects do not necessarily resemble each other. Hierarchical resemblance is also absent: negative determinates don't yield more similarity than their determinables. Objects that share the property not aqua are not necessarily more similar to each other than objects that share the property not blue. Nor does the reverse hold: two objects that have the property not blue are not necessarily more similar to each other than two objects that have the property not aqua. ${ }^{18}$

Fourth, incompatibility of determinates. A thing can't have the properties aqua and turquoise at the same time and at the same place, but it can have the properties not

\footnotetext{
16 One might hold that only that which has the potential to be blue can fail to be blue. According to this view, since the number 17 cannot be blue, it cannot fail to be blue in any meaningful sense. But such a move must be built into the analysis of the determinate/ determinable relationship, which Dowe does not. Thanks to an astute referee for articulating this view.

17 Nick Zangwill (2011) has also argued for the similar claim that negative properties have less determining power than positive properties.

18 One might be tempted by reverse resemblance, or the idea that more general negative properties such as not blue generate greater resemblances than not aqua. Since the property not aqua is entailed by the property not blue, the second pair will share all negated blue properties (not aqua, not navy, not turquoise, and so on). But negative properties do not generate genuine resemblance individually. For example, two objects that have the property not blue are not necessarily more similar than two objects that share the property not aqua with respect to the property not blue alone.
} 
aqua and not turquoise at the same time and in the same place. Similarly, a thing can't have the properties circular and square at once, but it can have the properties not circular and not square at once. Incompatibility of sibling determinates fails.

Now, one might concede that specificity, resemblance, and incompatibility do not apply to negative properties in these ways but nevertheless insist that mere satisfaction of entailment is sufficient to ground a determinate/ determinable relationship for negative properties. There are two major problems for such a claim. First: it casts its net too broadly. Having the property tallness entails the property being self-identical, even though being self-identical isn't a more specific way of being tall. Being a teacher entails being human, but being a teacher doesn't seem like a more specific way of being human. Second: when philosophers appeal to the determinate/ determinable relationship to do work for proportionality, more than mere entailment is required. Returning to Yablo's example: suppose that Sophie the bird is trained to peck at blue objects, and that she pecks at a turquoise object. Turquoise determines the properties blue, colored, and existent. But only blue and colored are plausible candidates for the cause of the pecking. Proportionality requires that the selection pool be multiple related properties at differing levels of specificity, not three unrelated properties. The other three features of the determinate/ determinable relationship are required to draw the appropriate pool of causal candidates.

Given the problem of irrelevant determining properties and the failure of three key components of the determinate/ determinable relationship-specificity, similarity, and incompatibility of determinates-- I conclude that the determinate/ determinable relationship does not apply to negative properties in its familiar form. As I shall now suggest, this result undermines Dowe's proportionality solution to the problem of profligate omissions.

\section{Two Problems For Proportionality about Omissions}

3.1 The Determinate/ Determinable Distinction Doesn't Apply to Negative Properties

First problem: the determinate/ determinable relationship doesn't hold between not receiving an influx of water and not receiving an influx of substance. The property not 
receiving an influx of water is not more or less specific than not receiving an influx of substance. Two things are not more similar in virtue of having the property not receiving an influx of water rather than the property not receiving an influx of substance. And an object or event can have the properties not receiving an influx of water and not receiving an influx of substance at once. Since the proportionality principle relies on negative properties rendering events differentially specific, a lack of differential specificity in negative properties blocks its application.

Despite the lack of a determinate/determinable relationship between properties, one might still counterfactually test individual properties for causal dependence apart from their relationship to each other. For example, one could ask: is it the case that the plant's dying counterfactually depends on its having the property not receiving an influx of water? But the counterfactual test makes no progress on the initial problem: indefinitely many specious counterfactual dependencies hold between the plant's death and individual properties, such as not being self-poisoning and not receiving an influx of substance from Barack Obama. The role of the apparatus of determinates and determinables is to answer the question of which counterfactuals are specious, and which are relevant to the causal story. Without this apparatus, we are simply facing the same question we had at the outset: which of the omissions that pass the counterfactual test are actually causes? Alone, the counterfactual test gives no extra non-causal explanation for the division between relevant and irrelevant omissions.

\subsection{Proportionality Treats the Wrong Problem}

Second problem: even if the proportionality strategy could overcome the challenges raised thus far, it would not answer the problem of profligate omissions that it purports to solve but, rather, an entirely different one. It purports to resolve causal profligacy at one level of detail, but instead, it resolves causal profligacy at different levels of detail-a concern distinct from the traditional problem of profligate omissions.

To see why, it will help to get a better grip on the problem of profligate omissions. Instances of the problem of profligate omissions have the form 's failure to had not occurred, the 
outcome would not have occurred."

This schema (hereafter: TP) draws from a pool of omissive causal candidates at the same level of causal detail for its satisfiers. In the plant example, the causal candidates for the plant's death are the gardener's failure to water the plant, Barack Obama's failure to water the plant, an artificially intelligent robot's failure to water the plant, and so on. Each candidate satisfies the TP schema "If 's failure to water the plant had not occurred, the plant's death would not have occurred." Since every TP schema includes the event type (a failure to water the plant) that led to the plant's death, its possible satisfiers already share the same level of detail. The traditional problem of profligate omissions involves culling the profligate satisfiers for TP. A desideratum for a satisfying solution is to give a criterion for selecting the gardener rather than Barack Obama as the cause of the plant's death.

Proportionality doesn't solve this problem, however. Rather, the problem it treats is different than the traditional problem of profligate omissions in two important ways.

First, the proportionality theorist works with a more general schema than TP. The proportionality theorist's schema has the form

(Profligacy*) "If had not possessed the property , the outcome would not have occurred."

Profligacy* (hereafter: $\mathrm{P}^{*}$ ) differs from TP in several important respects. First, due to its structure, it selects from a pool of causal candidates at different levels of detail. Possible satisfiers for $\mathrm{P}^{*}$ are properties of the plant like not receiving an influx of substance, not receiving an influx of water, not receiving an influx of water from the gardener, not receiving an influx of water from the gardener while humming, and so on. The proportionality theorist individually tests these properties for satisfaction of the broader counterfactual schema "If the plant had not possessed the property __, the plant would not have died." Because this schema is broader than the traditional profligate omissions schema, it naturally opens the pool of causal candidates to properties at different levels of detail. Proportionality selects a satisfier for $\mathrm{P}^{*}$, but not for TP.

To make this idea clearer, consider another example. Suppose that Bystander, the 
only person in the physical vicinity of Victim, sees Victim stop breathing. Bystander knows the Heimlich maneuver, but doesn't administer it, and Victim dies. Intuitively, Bystander is a cause of Victim's death.

The TP schema for this case is "If___ 's failure to administer the Heimlich maneuever hadn't occurred, Victim would not have died." The possible satisfiers for the TP schema are Bystander, Barack Obama, a life-saving robot, and so on. Though Victim's death is counterfactually dependent on each omission, the death is not, intuitively, caused by the latter two omissions.

But proportionality neither vindicates this idea nor relieves this kind of profligacy. Rather, the proportionality strategist generates the following $\mathrm{P}^{*}$ schema: "If Victim had not possessed the property ___ Victim would not have died." The possible satisfiers for this $\mathrm{P}^{*}$ schema are differentially detailed properties of Victim, such as:

Failure to have pressure exerted on the upper respiratory system Failure to have pressure exerted on the upper respiratory system by a person Failure to have pressure exerted on the upper respiratory system by Bystander ....and so on. According to proportionality, Victim's possession of the property failure to have pressure exerted on the upper respiratory system is the cause of his death. But the proportionality strategy ignores the type of profligacy generated by the TP schema: the various entities at the same level of detail (Bystander, Barack Obama, a life-saving robot) that failed to administer the Heimlich maneuver. The strategy is silent on the problem it purports to solve-profligate omissions at the same level of detail-and instead solves the problem of profligate causes at different levels of detail. This is $a$ problem, but not the traditional problem of profligate omissions.

The second way the proportionality theorist's target differs from the traditional problem of profligate omissions is that its causal candidates are metaphysically different: whereas TP draws from omissive events, $\mathrm{P}^{*}$ draws from a pool of properties of the effect itself. This difference is represented by another dissimilarity between TP and P*. Recall that TP has the structure "If 's failure had not occurred, then would not have occurred." In the example of the plant's death, the candidate satisfiers for the schema are omissive causes external to the effect: the gardener's failure, Barack Obama's 
failure, and so on. But candidate satisfiers for $\mathrm{P}^{*}$ are properties of the plant: satisfiers of the schema "If the plant had not possessed the property , the plant would not have died." The candidate properties of the plant are not receiving an influx of water, not receiving an influx of substance, and so on. The proportionality strategist replaces the problem of selecting a particular salient omissive cause with the problem of selecting a particular negative property of the effect. The proper satisfier of the $\mathrm{P} *$ schema is a negative property not $x$ such that had the effect had the property $x$, the outcome would not have occurred. Proportionality solves that problem instead.

This difference is important because the result is that any omissive causal candidate external to the effect in question is not its cause. Proportionality selects a negative property of the plant as the cause of its death. But negative properties of the plant are not omissive causes of the type generated by the problem of profligate omissions. Not only does proportionality deny that the gardener caused the plant to die; it also denies that anything but a feature of the plant itself-its possession of the property failure to receive an influx of substance-caused the plant to die. ${ }^{19}$ Though proportionality for omissions is already radical due to its denial of the gardener's causal role in the plant's death, this result is more radical than previously thought: it suggests that there isn't causation by omission of the familiar sort at all. For only a property of the plant itself, rather than an omissive event external to the plant, caused its death. Similarly for Victim's death: strictly speaking, no omissive event external to him caused his death. Rather, his own failure to have the property pressure exerted on the upper respiratory system caused his demise.

This is not only a counterintuitive result for causation, but is disastrous for dependent projects like the semantics of causation and moral responsibility. A suitable semantics of causation can't be given if claims like "The gardener's failure to water the plant caused the plant to die" and "Bystander's negligence caused Victim to die" aren't true.

Nor can an account of moral responsibility function with such a result. In the

${ }^{19}$ A referee points out that this problem is an instance of the problem of proximate versus remote causes: something external to and causally remote from the plant causes the plant to have the negative property having failed to receive an influx of water. But the remote cause cannot be traced specifically to the gardener, because many people caused the plant to have the negative property having failed to receive an influx of water, including, for example, Barack Obama. Thus the problem of profligacy reemerges with remote causes. 
Bystander example, proportionality selects Victim's property failure to have pressure exerted on the upper respiratory system as the cause of death. That property is held by Victim himself. It is thus false that anyone or anything external to him caused his death. This radical lesson generalizes to every case of putative causation by omission: nothing is ever strictly caused by anything external to the effect. Proportionality for omissions generates a world of self-caused outcomes, but not causation by omission per se. ${ }^{20}$

One might object that the problem of profligate omissions has been solved by eliminating unnecessary detail in the relevant counterfactuals. But the result of proportionality for omissions is unhelpful: it both changes the problem and eliminates causation by omission between distinct objects entirely. Such an elimination does not advance the debate on profligate omissions so much as repudiate its central desiderata.

\section{Conclusion}

Dowe's proportionality strategy for omissions faces several difficulties. First, it relies on the determinate/ determinable relationship to address profligate causation by omission. However, negative properties don't exhibit three key features of the determinate/ determinable relationship, and mere entailment doesn't suffice for the metaphysical work that the relationship must do. Since omissions essentially involve negative properties, proportionality is not a viable solution to the problem of profligate omissions. Further, proportionality treats a different problem of profligacy than the one it purports to solve. The cost of proportionality about omissions - that there is no causation by omission at all-outweighs the possible benefits of such a view.*

\section{REFERENCES}

Dowe, P. 2010, Proportionality and Omissions. Analysis 70 (3): 446-451.

\footnotetext{
20 The proportionality theorist might respond that external causation enters the picture when, e.g., the gardener causes the plant to have the property failure to receive an influx of water. Thus the failure to receive an influx of water is a relational property that holds between the plant and the gardener. But profligate omissions (the gardener's, Barack Obama's, an artificially intelligent robot's) cause the plant to have this property, and so this is no improvement on the traditional problem of profligate omissions.

* Thanks to Mark Balaguer, Helen Beebee, Phil Dowe, Lewis Powell, David Pitt, Kate Ritchie, Alex Rosenberg, Carolina Sartorio, Larry Shapiro, and Jessica Wilson for valuable feedback on this paper.
} 
FunkHouser, E. 2006, The Determinate-Determinable Relation. Noûs 40 (3):548-569.

Miller, K, Colthart, R. Baron, S. Majeed, M. 2013, "What is a Negative Property?" Philosophy. 88(1): 33-54.

SARTORIO, C. 2010, The Prince of Wales Problem for the Counterfactual Theory of Causation. New Waves in Metaphysics, ed. Allan Hazlett. Palgrave Macmillan, New York, pp. 259-2761

SHAPIRO, L. AND Sober, E. Against Proportionality. Analysis 2012, 72 (1): 89-93.

WeSlaKe, B. 2013, Proportionality, Contrast, and Explanation. Australasian Journal of Philosophy. vol. 91, no. 4, pp. 785-797.

WILSON, J. 2012, Fundamental Determinables. Philosopher's Imprint.

YaBlo, S. 1992, Mental Causation, Philosophical Review 101: 245-80.

ZANGWILL, N. 2011, Negative Properties. Nous 45:3 (2011) 528-556. 